\title{
Optimization Control of Motor Vehicle at Intersection Based on Green Building Materials
}

\author{
Zijun LIANG ${ }^{\mathrm{a}, \mathrm{b}, 1}$, Xuejuan ZHAN,a, ${ }^{\mathrm{a}}$, Wei KONG ${ }^{\mathrm{a}, \mathrm{b}}$, Wei LIU ${ }^{\mathrm{a}}$, Yuanyuan $\mathrm{ZHAO}^{\mathrm{a}}$, \\ Yingying $\mathrm{GAO}^{\mathrm{a}}$, Yiran $\mathrm{NIU}^{\mathrm{a}}$ and Jingjing $\mathrm{QI}^{\mathrm{c}}$ \\ a School of Urban Construction and Transportation, Hefei University, China \\ ${ }^{\mathrm{b}}$ Anhui Province Transportation Big Data Analysis and Application Engineering \\ Laboratory, China \\ ' Anhui Dar intelligent control system stock co., LTD, China
}

\begin{abstract}
Along with the rapid development of urban social economy, under the influence of sustainable development strategy, urban road traffic engineering design should adhere to the concept of environmental protection and develop towards greening and modernization. Firstly, this method uses the green environmental construction materials, such as hot-melt reflective marking paint, color asphalt concrete pavement and polyurethane elastic isolation column, to build green traffic spaces at intersection; Then on this basis, the motor vehicles signal optimization technology is used to balance the space-time resource allocation of the motor vehicle traffic flow, motor vehicle traffic optimal phase combination analysis method is put forward, and the optimal calculation of motor vehicle traffic time is optimized by the signal timing optimization method that considers the overlapping phase. Finally, through the evaluation and analysis of the VISSIM traffic simulation software, the results show that the method based on green building materials has effectively defined about the green traffic spaces of motor vehicles, and under this premise, the efficiency of motor vehicle traffic is further improved by signal optimization technology, providing the foundation for the research on green traffic on urban roads.
\end{abstract}

Keywords. Green building materials, motor vehicle, green traffic space, signal optimization method, VISSIM traffic simulation.

\section{Introduction}

With the rapid development of urban social economy, the urban motorization level is rapidly improved, problems such as unreasonable design of intersection channelization and inaccurate signal timing are the most common [1]. As the main body of traffic management, in recent years, many scholars at home and abroad have made relevant research on the access control such as motor vehicle for intersection channelization phase and timing design. In terms of channelization design, Song Bowen has put forward targeted strategy for the design of urban road intersection channelization design [2]. Yan

${ }^{1}$ Corresponding Author, Zijun LIANG, School of Urban Construction and Transportation, Hefei University, China, Anhui Province Transportation Big Data Analysis and Application Engineering Laboratory, China; E-mail: 953609319@qq.com. 
Min have elaborated the basic principles of channelization design of urban road intersections [3]. Zhang Peng have analyzed the design of intersection channelization from aspects of motorized lane, non-motorized lane and pedestrian crossing, and put forward some methods and measures to ensure the traffic safety and traffic efficiency of intersection vehicles and pedestrians [4]. In terms of phase design, in order to achieve minimum intersection traffic delay, Zheng [5] and Barnes [6] and others have studied the optimal phase sequence at the intersection, when two signal phases collide, the phase of higher traffic flow (high demand) is given priority. Zhang Leiyuan et al. have used the four-stage method to design the intersection signal phase [7]. Gao Yunfeng et al. have expounded the general design principles of the signal phase at the intersection and optimized the release order between the left turn and the straight phase to minimize vehicle delays [8]. In terms of timing optimization, the initial study dates back to 1958, when Webster first obtained the best cycle formula by minimizing total vehicle delays at intersections [9]. Wang, based on the optimization principle of minimizing vehicle exhaust emissions and road network impedance, have studied the two-stage optimization model of travel matrix estimation and system equilibrium allocation, and solved it by using particle swarm algorithm [10]. Qu Qiuwen has used simulation software to optimize the adjustment and comparative analysis of the intersection timing scheme, and given the optimal timing scheme of the intersection and put forward some related suggestions [11]. With the deepening of the concept of green commuting, the use of green environmental materials has become the inevitable trend of road traffic design and construction development, so the application of environmental protection materials in traffic optimization design emerged [12], Nie Sheng has studied the application of environmental protection materials in road traffic engineering [13], Liu Zhixian has studied the application of a new type of green environmental protection materials in highway engineering [14], however, the current research in this aspect is still less, especially the green building materials in the intersection of motor vehicles in the optimization design has not been mentioned in the literature.

Therefore, this paper is written for the intersection motor vehicle travel, with green traffic as the concept, from the two dimensions of traffic space and time to consider, this paper puts forward the intersection motor vehicle traffic optimization control method based on the green building materials. First of all, use more environmentally protection green building materials in the optimization of transportation facilities, and construct motor vehicle green traffic space at intersection; Then in the aspect of signal optimization, the optimal combination analysis method of motor vehicle traffic phase is proposed, and the effective passage time of intersection motor vehicle is optimized by the signal timing optimization method considering the overlapping phase. Finally, through the actual intersection case design and simulation analysis, the rationality and superiority of the method in this paper are verified.

\section{Application of Green Building Materials in Motor Vehicle Traffic Facilities Design}

According to the guidelines of the Sustainable Development in China, using environmental protection materials has become an inevitable trend of construction development. The application of environmental protection materials at this stage reflects the green environmental protection performance of traffic engineering to a large extent [15]. Therefore, this paper mainly uses transportation facilities made of hot-melt 
reflective marking paint, color asphalt concrete, elastic isolation column and isolation guardrail and other green building materials, for channelization design of the existing intersection of motor vehicle green traffic space.

The main component of hot-melt reflective marking paint is resin. The coating is firmly integrated with the road surface, has strong reflectivity with good visibility during the day and night, and has slip resistance and wear resistance to ensure driving safety and service life [16].

The color asphalt concrete refers to the decolorized asphalt mixed with various color stone, color materials and additives and other materials at a specific temperature, and then after paving, grinding, a certain strength and road performance of the road material is formed, mainly playing the function of traffic induction, it has strong discernability so that pedestrians focus. Strong anti-aging ability, high temperature stability and good durability, and not easy to deformation, peeling and other phenomena, and the grassroots bonding is good [17]. Among them, asphalt components mainly use rubber powder modified asphalt, crumb rubber powder modified asphalt is made of $20 \%$ rubber powder (processed from used tires) and 80\% asphalt processing [18]. It can improve the service life of the road surface, reduce noise, reduce vibration, improve thermal stability and thermal cracking, improve anti-icing property. In current China, its application of highway pavement engineering is becoming more and more common, showing many advantages [19].

The elastic isolation column cylinder is made of TPU (polyurethane) material, is a binary or multiple isocyanate and binary or multiple hydroxyl compounds of polymeric materials, which can be used in the manufacture of plastics, wear-resistant synthetic rubber, synthetic fibers, hard and soft foam, adhesives and coatings, etc., widely used in the transportation industry [20]. It has good elasticity, it can withstand the external force of the violent impact quickly restored to its original condition; has a good reflective performance, clearly sketching the road outline; effectively isolate among the city intersection pedestrian walk, non-motorized lanes and motorized vehicle lanes, to remind and alert the driving vehicles, once hit will not cause secondary injury; And the installation is more convenient, with the internal expansion screw to secure it on the ground, the installation is secure.

\section{Channelization Design of Green Traffic Space for Motor Vehicle}

Intersection motor vehicle traffic should give priority to the problem of traffic capacity matching, the second is to induce vehicles to follow each other's paths, in addition to reducing the interference of non-motor vehicles for motor vehicles, to ensure the traffic safety. The reflective markers of green building materials, color pavement, isolation column and other channelizing design can be used to clarify the green traffic space of motor vehicles. Combined with the existing literature, the specific measures proposed in this paper mainly include the following points.

(1) Inlet and outlet traffic capacity matching design at intersection

Mismatching the traffic capacity of the intersection's inlet and outlet often results in vehicle queue congestion. In view of the phenomenon of the mismatch in the number of lanes, the method of channelizing is to change the nature of lane traffic, thereby reducing the number of inlet lanes or increasing the number of outlet lanes. The hot-melt reflective marking paint can be used to lay the marking lines, through good visual effect to guide the vehicle in and out of the intersection. 
(2) The internal diversion lines design at intersection

The design of the internal diversion lines at the intersection is determined by determining the traffic trace and turning radius of each vehicle in each traffic lane. On the basis of determining the diversion lines, the straight or left turn waiting area of the motor vehicle is determined, taking into account the existence of the collision point and the safety of the vehicle, the parking line of the waiting area is set at a certain safe distance from the track line of the vehicle in the previous phase. The marking lines can be laid with hot-melt reflective marking paint, and the vehicle waiting area can be paved yellow with color asphalt concrete pavement materials.

(3) The traffic space design for barrier

Non-motorized vehicles often cause greater interference to motor vehicles, so it is necessary to isolate non-motor vehicles and motor vehicles in space, eliminating nonconflict points. First of all, the color asphalt concrete pavement materials will be paved the non-motorized lanes in green, the diversion island paved in red, wherein the asphalt material is crumb rubber powder modified asphalt. Then the polyurethane elastic isolation column is used to physically isolate the motorized lanes and the non-motorized lanes. Finally, the diversion line is used to reserve the non-passable space inside the intersection, which provides the guarantee for regulating the non-passable order of the machine.

\section{Signal Optimization Technique of Motor Vehicle Traffic}

\subsection{Phase Optimal Combination Analysis of Motor Vehicle Traffic}

Suppose that the main control flow direction is $a_{i}(\mathrm{i}=1,2, \ldots, 8)$, as shown in figure 1 , which is the east straight, east left, south, south left, west, west, north, and north left, where the right turn is not controlled that should be ignored. As shown in table 1, the lane flow ratio corresponding to $a_{i}$ is $y_{i}$, calculation formula is shown in equation (1), $a_{i}$ can form 9 different phase schemes, of which group 1 to group 4 is a conventional phases, group 5 to 9 is a phase scheme containing overlapping phase, wherein the overlapping phase refers to the two phases between straight forward and left, Embedding a transition phase in which a one-way straight line is released at the same time as a left turn is suitable for uneven distribution of traffic at intersections. $Y_{j}(\mathrm{j}=1,2, \ldots, 9)$ is the flow ratio in a cycle for the nine phase combinations described above [21], as shown in table 1 . The phase combination for table 1 is shown in figure 2 .

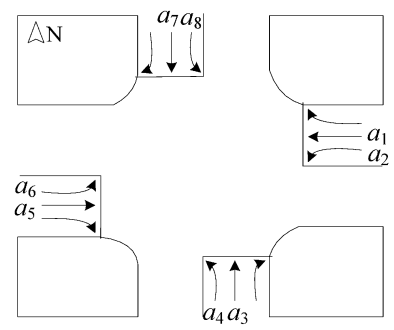

Figure 1. Main control flow at intersection. 
Table 1. Phase combination scheme and calculation of flow ratio in a cycle

\begin{tabular}{|c|c|c|c|c|c|}
\hline No. & \multicolumn{4}{|c|}{ Phase combination } & $\boldsymbol{Y}_{j}$ \\
\hline 1 & $a_{1}, a_{5}$ & $a_{2}, a_{6}$ & $a_{3}, a_{7}$ & $a_{4}, a_{8}$ & $Y_{1}=\max \left(y_{1}, y_{5}\right)+\max \left(y_{2}, y_{6}\right)+\max \left(y_{3}, y_{7}\right)+\max \left(y_{4}, y_{8}\right)$ \\
\hline 2 & $a_{1}, a_{5}$ & $a_{2}, a_{6}$ & $a_{3}, a_{4}$ & $a_{7}, a_{8}$ & $Y_{2}=\max \left(y_{1}, y_{5}\right)+\max \left(y_{2}, y_{6}\right)+\max \left(y_{3}, y_{4}\right)+\max \left(y_{7}, y_{8}\right)$ \\
\hline 3 & $a_{1}, a_{2}$ & $a_{5}, a_{6}$ & $a_{3}, a_{7}$ & $a_{4}, a_{8}$ & $Y_{3}=\max \left(y_{1}, y_{2}\right)+\max \left(y_{5}, y_{6}\right)+\max \left(y_{3}, y_{7}\right)+\max \left(y_{4}, y_{8}\right)$ \\
\hline 4 & $a_{1}, a_{2}$ & $a_{5}, a_{6}$ & $a_{3}, a_{4}$ & $a_{7}, a_{8}$ & $Y_{4}=\max \left(y_{1}, y_{2}\right)+\max \left(y_{5}, y_{6}\right)+\max \left(y_{3}, y_{4}\right)+\max \left(y_{7}, y_{8}\right)$ \\
\hline 5 & \multicolumn{2}{|c|}{$a_{1}, a_{2}, a_{5}, a_{6}$} & $a_{3}, a_{7}$ & $a_{4}, a_{8}$ & $Y_{5}=\max \left(y_{1}+y_{6}, y_{2}+y_{5}\right)+\max \left(y_{3}, y_{7}\right)+\max \left(y_{4}, y_{8}\right)$ \\
\hline 6 & \multicolumn{2}{|c|}{$a_{1}, a_{2}, a_{5}, a_{6}$} & $a_{3}, a_{4}$ & $a_{7}, a_{8}$ & $Y_{6}=\max \left(y_{1}+y_{6}, y_{2}+y_{5}\right)+\max \left(y_{3}, y_{4}\right)+\max \left(y_{7}, y_{8}\right)$ \\
\hline 7 & $a_{1}, a_{5}$ & $a_{2}, a_{6}$ & \multicolumn{2}{|c|}{$a_{3}, a_{4}, a_{7}, a_{8}$} & $Y_{7}=\max \left(y_{1}, y_{5}\right)+\max \left(y_{2}, y_{6}\right)+\max \left(y_{3}+y_{8}, y_{4}+y_{7}\right)$ \\
\hline 8 & $a_{1}, a_{2}$ & $a_{5}, a_{6}$ & \multicolumn{2}{|c|}{$a_{3}, a_{4}, a_{7}, a_{8}$} & $Y_{8}=\max \left(y_{1}, y_{2}\right)+\max \left(y_{5}, y_{6}\right)+\max \left(y_{3}+y_{8}, y_{4}+y_{7}\right)$ \\
\hline 9 & \multicolumn{2}{|c|}{$a_{1}, a_{2}, a_{5}, a_{6}$} & \multicolumn{2}{|c|}{$a_{3}, a_{4}, a_{7}, a_{8}$} & $Y_{9}=\max \left(y_{1}+y_{6}, y_{2}+y_{5}\right)+\max \left(y_{3}+y_{8}, y_{4}+y_{7}\right)$ \\
\hline
\end{tabular}

Among them, $y_{i}$ is the lane flow ratio of traffic flow to $a_{i}, q_{i}$ and $s_{i}$ are the average lane flow $(\mathrm{pcu} / \mathrm{h})$ and lane saturation rate $(\mathrm{pcu} / \mathrm{h})$ respectively.

The signal cycle duration can be calculated by the Webster cycle duration formula. Calculation formula is shown in equation (2).

$$
C=\frac{1.5 L+5}{1-Y_{j}}
$$

Where $\mathrm{C}$ is the signal cycle length (s), $\mathrm{L}$ is the total signal loss time (s).

As can be seen from the $Y_{j}$ calculation in table 1 , the value of the different phase combinations corresponding to $Y_{j}$ is determined by the maximum value of $y_{i}$ in conventional phase and the maximum value of the sum of turn straight and left in combination phase. The greater difference between the $y_{i}$ in each phase, the larger $Y_{j}$ and the larger $\mathrm{C}$, resulting in increased signal control delay. Therefore, the scheme with smallest value of $Y_{j}$ can be chosen as the optimal phase combination scheme, and the phase combination corresponding to table 1 as be shown in figure 2 .

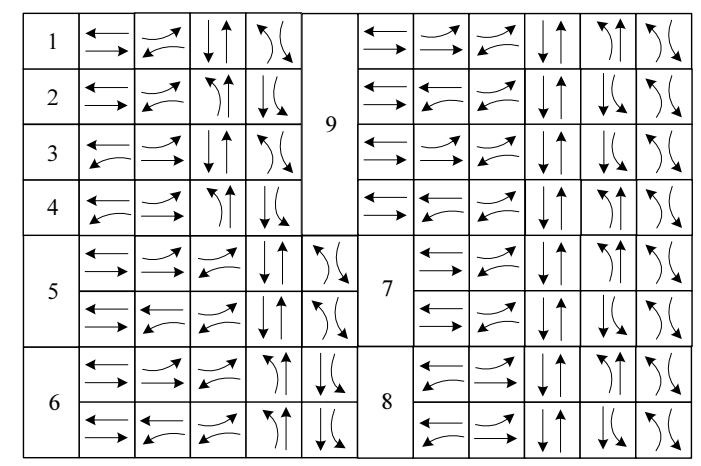

Figure 2. Control flow direction and phase combination scheme. 


\subsection{Overlapping Phase Signal Timing Design of Motor Vehicle}

According to the lane flow ratio $y_{i}$ to draw the conventional phase and overlapping phase signal control chart as shown in figure 3, through comparison, it can be found that in conventional phase, each direction of flow controlled $a_{i}$ of phase $k$ to a traffic flow release time is synchronous start and synchronous end, in the case of flow rate is not equal, it is easy to produce a direction of controlled $\mathrm{a}_{\mathrm{i}}$ a traffic flow empty with low traffic efficiency. Compared with the conventional phase, the overlapping phase allocates the traffic time according to the traffic proportion, and the traffic efficiency is higher.

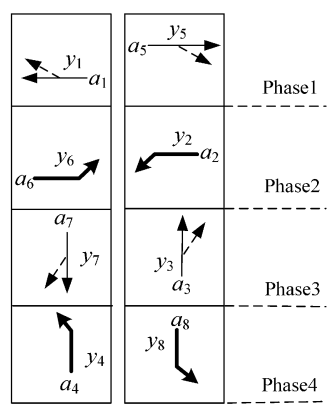

(a)Conventional phase

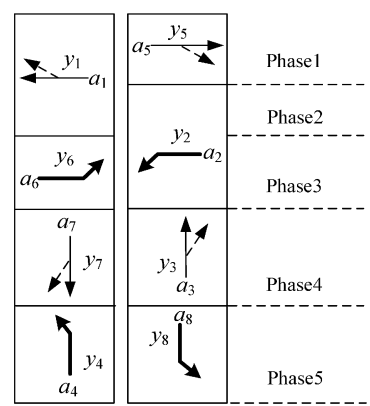

(b) Overlapping phase

Figure 3. Signal control chart of conventional phase and overlapping phase.

Taking the overlapping phase shown in figure ( $3 \mathrm{~b})$ as the optimal phase combination, the calculation process of signal timing is illustrated. $\mathrm{y}_{1}>\mathrm{y}_{5}, \mathrm{y}_{2}>\mathrm{y}_{6}, \mathrm{y}_{2}+\mathrm{y}_{5}>\mathrm{y}_{1}+$ $\mathrm{y}_{6}, \mathrm{y}_{7}>\mathrm{y}_{3}, \mathrm{y}_{8}>\mathrm{y}_{4}$, it is in accordance with the fifth case in table 1 .

First, calculate the lane flow ratio $y_{i}$ of each traffic flow $a_{i}$, and the calculation formula is shown in equation (1) above.

Then according to the formula shown in the fifth case of table 1, calculate the flow ratio in a cycle $Y_{5}$.

Secondly, calculate the total signal loss time of a cycle, and the calculation formula is shown in equation (3).

$$
L=\sum\left(L_{k}+r_{k}\right)
$$

Among them, $\mathrm{L}_{\mathrm{k}}$ is the start-up loss time of phase $\mathrm{k}(\mathrm{s}), \mathrm{r}_{\mathrm{k}}$ is the full red time of phase $\mathrm{k}(\mathrm{s})$.

Then using the Webster cycle duration formula to calculate the signal duration of a cycle. The calculation formula is shown in equation (2) above.

Finally, determine the total effective green time $G_{E}$ of a cycle and effective green time of each phase $\mathrm{g}_{\mathrm{E}}^{(\mathrm{k})}$.

The signal timing calculation formulas of overlapping phase are shown in equation (4) to (10).

$$
G_{E}=C-L
$$




$$
\begin{gathered}
g_{E}^{(1)}=G_{E} \times \frac{y_{5}+y_{2}}{Y} \times \frac{y_{5}}{y_{5}+y_{2}} \\
g_{E}^{(1+2)}=G_{E} \times \frac{y_{5}+y_{2}}{Y} \times \frac{y_{1}}{y_{1}+y_{6}} \\
g_{E}^{(2+3)}=G_{E} \times \frac{y_{5}+y_{2}}{Y} \times \frac{y_{2}}{y_{5}+y_{2}} \\
g_{E}^{(3)}=G_{E} \times \frac{y_{5}+y_{2}}{Y} \times \frac{y_{6}}{y_{1}+y_{6}} \\
g_{E}^{(4)}=G_{E} \times \frac{y_{7}}{Y} \\
g_{E}^{(5)}=G_{E} \times \frac{y_{8}}{Y}
\end{gathered}
$$

Among them, $G_{E}$ is the total effective green time $(s), g_{E}^{(k)}$ is the effective green time of the phase $\mathrm{k}(\mathrm{s})$.

\section{Design example}

\subsection{Canalization Design at the Intersection of Beijing Road and Huangshan Road}

The intersection is a deformity intersection, and belongs to the $\mathrm{X}$-shaped intersection in the classification. The current channelization diagram is shown in figure 4.

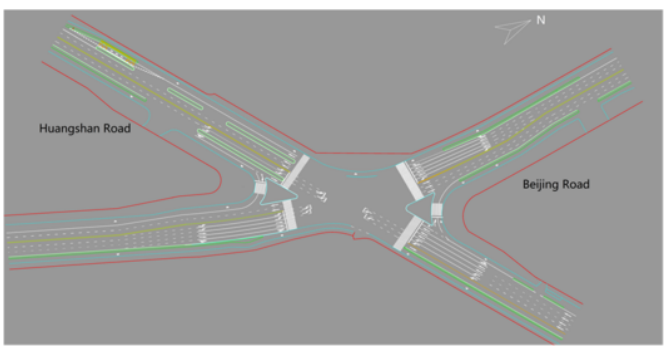

Figure 4. Current channelization diagram of Beijing road and Huangshan road intersection.

According to the method proposed in this paper, the green traffic space is designed by channelization, mainly including the following points.

(1) Inlet and outlet traffic capacity matching design at intersection

The north inlet lane of the intersection has three lanes, the south outlet lane has two lanes, leading to the mismatched traffic capacity and congestion at the south exit easily. The survey found that the straight traffic volume of the north inlet lane is larger and the straight traffic volume of the south outlet lane is relatively less, so the number of inlet lanes in the south is reduced to increase the number of outlet lanes by 3 , meeting the requirements of the matching traffic capacity.

(2) The internal diversion lines design at intersection

The passage distance of the intersection is too long to pass, the intersection traffic space is narrow, resulting in serious motor vehicles interweaving. By setting the 
diversion lines and the straight and left-turn waiting area, shorten the distance of the motor vehicles through the intersection, guide the motor vehicles to travel according to the prescribed trace, use environmental protection type of hot-melt reflective marking paint to lay the diversion lines, and use color asphalt concrete pavement material to lay the waiting area in yellow.

(3) The traffic space design for barrier

The north-south exit of the intersection has no non-motorized lanes, resulting in the interweaving of the motor vehicles and non-motor vehicles traffic and greater safety potential hazard. First, increase non-motorized lanes in the south and north exits, and then use color asphalt concrete pavement materials to pave the non-motorized lanes pavement of the intersection in green; Secondly, the color asphalt concrete pavement materials are used to lay the diversion island in red; Finally, use polyurethane elastic isolation column as physical barriers to isolate the traffic lanes and diversion island.

The optimized channelization diagram is shown in figure 5 .

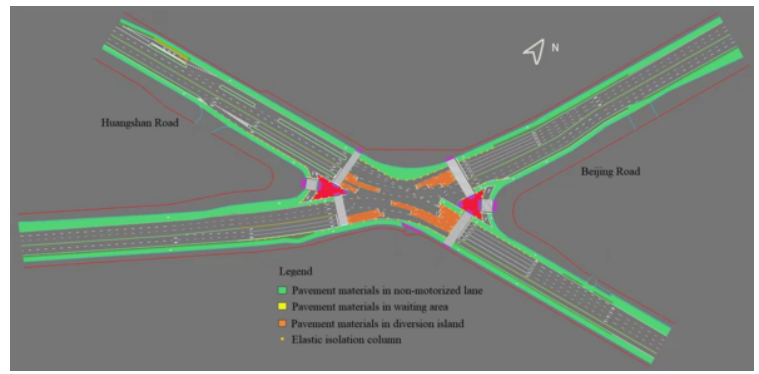

Figure 5. Optimized channelization diagram of Beijing road and Huangshan road intersection.

\subsection{Overlapping Phase Signal Timing Design at the Intersection of Beijing Road and Huangshan Road}

Select the morning peak hour when the traffic volume is larger from 07:30 to 08:30 at the intersection of Beijing Road and Huangshan Road, and the signal phase matching design is carried out by using the method in this paper, and the traffic flow data of the intersection are shown in table 2. Among them, phase scheme $\mathrm{A}$ is the conventional fourphase based on current channelization, phase scheme B is the overlapping phase based on optimization channelization, as be shown in figure 6 . The signal timing scheme is shown in table 3.

Table 2. Average hourly traffic flow data from $07: 30$ to $08: 30$ at the intersection

\begin{tabular}{cccccc}
\hline direction & $\begin{array}{c}\text { Flow } \\
\text { direction }\end{array}$ & $\begin{array}{c}\text { Total flow } \\
\text { (pcu/h) }\end{array}$ & Number of lanes & $\begin{array}{c}\text { average lane } \\
\text { flow }\end{array}$ (pcu/h) & $\begin{array}{c}\text { lane saturation } \\
\text { rate(pcu/h) }\end{array}$ \\
\hline \multirow{2}{*}{ East } & straight & 696 & 3 & 232 & 1871 \\
& left & 552 & 2 & 276 & 1767 \\
\multirow{2}{*}{ West } & straight & 96 & 1 & 96 & 1880 \\
& left & 326 & 2 & 163 & 1863 \\
\multirow{2}{*}{ South } & straight & 1240 & 2 & 620 & 1860 \\
& left & 222 & 1 & 222 & 1761 \\
\multirow{2}{*}{ North } & straight & 1480 & 3 & 493 & 1908 \\
& left & 152 & 1 & 152 & 1861 \\
\hline
\end{tabular}




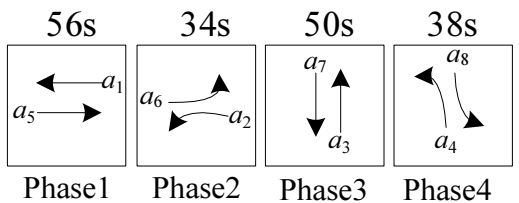

(a) Phase scheme A

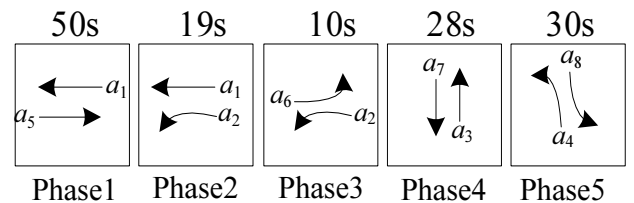

(b) Phase scheme B

Figure 6. Phase scheme design.

Table 3. Signal timing scheme

\begin{tabular}{ccccccccccc}
\hline \multirow{2}{*}{ No. } & \multirow{2}{*}{ Phase scheme } & \multirow{2}{*}{ Cycle(s) } & & \multicolumn{10}{c}{ Timing(s) } \\
\cline { 4 - 11 } & & & $\boldsymbol{a}_{\mathbf{1}}$ & $\boldsymbol{a}_{\mathbf{2}}$ & $\boldsymbol{a}_{\mathbf{3}}$ & $\boldsymbol{a}_{\mathbf{4}}$ & $\boldsymbol{a}_{\mathbf{5}}$ & $\boldsymbol{a}_{\mathbf{6}}$ & $\boldsymbol{a}_{\mathbf{7}}$ & $\boldsymbol{a}_{\mathbf{8}}$ \\
\hline 1 & $\mathrm{~A}$ & 178 & 56 & 34 & 50 & 38 & 56 & 34 & 50 & 38 \\
2 & $\mathrm{~B}$ & 137 & 69 & 29 & 28 & 30 & 50 & 10 & 28 & 30 \\
\hline
\end{tabular}

\section{VISSIM Analysis}

The phase scheme A, B corresponding to the channelization diagram and signal timing scheme are introduced into the VISSIM traffic simulation model. The current simulation model is shown in figure 7 , the optimization simulation model is shown in figure 8 , the simulation model runs with the traffic flow data of table 2 in 3600s, comparing and analyzing the traffic efficiency of scheme $\mathrm{A}$ and $\mathrm{B}$, the corresponding analysis results of the different schemes are shown in figure 9.

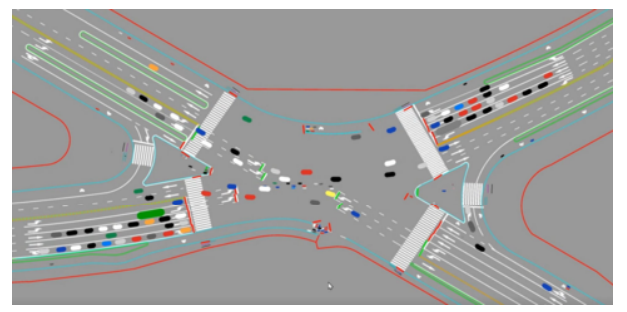

Figure 7. Current simulation model.

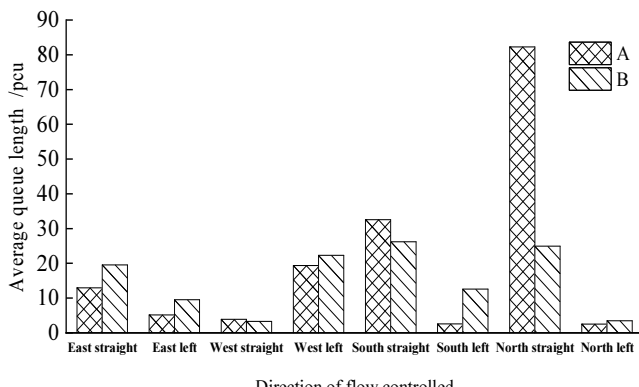

(a)Average queue length

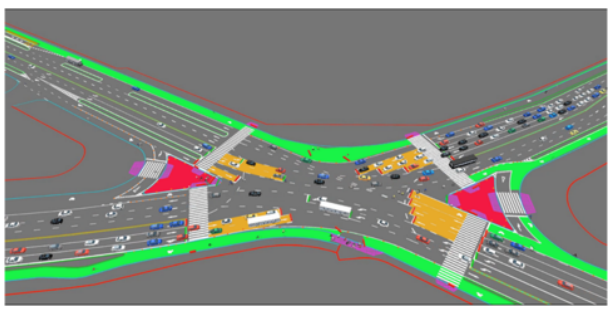

Figure 8. Optimized simulation model.

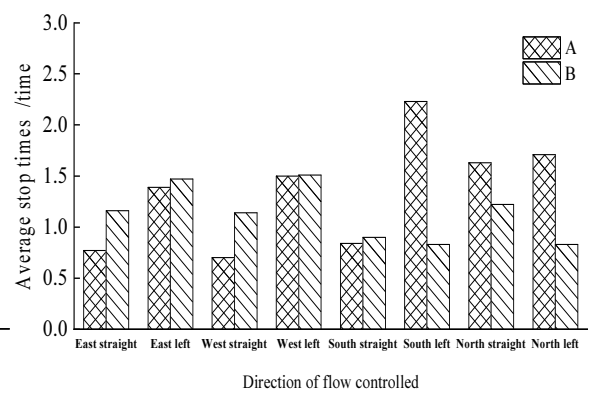

(b) Average stop times 


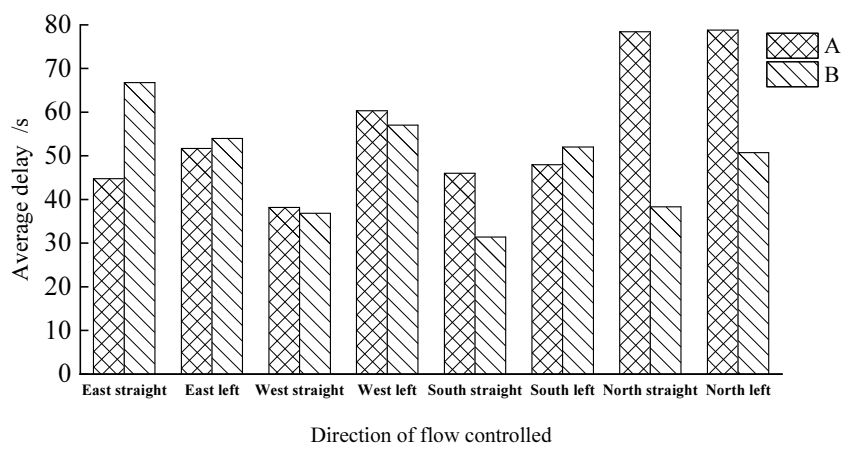

(c) Average delay

Figure 9. Traffic simulation analysis results of different schemes.

In figure 9(a), the average queue length of scheme B is significantly lower than that of scheme A, with an average reduction by $23.6 \%$. The reason is that by matching the traffic capacity of north-south inlets and outlets, congestion queues at south exits is reduced. At the same time, by adding straight and left turn waiting areas at the same time to enable motor vehicles to enter the intersection ahead of time, shortening the length of the inlet queue.

In figure 9(b), the average number of vehicles stop times in scheme B is lower than in scheme A, with an average reduction of 1.1 times. The reason is that through nonphysical barriers of the machine, the number of stop times between motor vehicles and non-motor vehicles decreases in the number of conflict points within a unit of time.

In figure 9(c), the average vehicle delay in scheme B is significantly lower than in scheme A, with an average reduction by $23.76 \%$. The reason is that the traffic flow distribution at the intersection is not balanced, and scheme B optimizes the signal phase matching after using this method in the paper, the cycle length is less than scheme A, which improves the vehicle traffic efficiency and reduces the vehicle delay in the signal cycle.

\section{Conclusion}

This paper uses green building materials to construct the green traffic space of motor vehicle at intersection, then puts forward the optimal phase combination analysis method and the signal timing optimization method of overlapping phase, and finally, with the actual intersection as the case, the channelization design of motor vehicle traffic space based on green building materials and the signal timing optimization scheme of overlapping phase are completed, and the effectiveness of this method is verified by VISSIM simulation software.

\section{Acknowledgments}

This work was supported by Talent Research Fund Project of Hefei University (grant no. 18-19RC03), University Natural Sciences Research Project of Anhui Province (grant no. KJ2020A0663) and General Project of Anhui Natural Science Foundation in 2021 (grant no. 2108085ME189). 


\section{References}

[1] Liu Deng. Phase Design and Signal Timing for an Isolated Intersection [D]. North China University of Technology, 2021.

[2] Song Bowen, Wang Jichang, Xu Jiacheng. Discussion on Design of City Road Intersection Channelization [J]. Shandong Transportation Technology,2021(01):127-128.

[3] Yan Min. Key points on Design of City Road Intersection Channelization and Instance Analysis [J]. Sichuan Cement,2020(07): 89-90.

[4] Zhang Peng. Discussion on Design of City Road Intersection Channelization [J]. HEILONGJIANG JIAOTONG KEJI,2020,43(05): 6-7.

[5] Zheng G, Xiong Y, Zang X, et al. Learning phase competition for traffic signal control[C]/Proceedings of the 28th ACM International Conference on Information and Knowledge Management.2019: 1963-1972.

[6] Barnes J, Paruchuri V. Optimal phase ordering of traffic signals to reduce stopped delay[C]//2012 IEEE 26th International Conference on Advanced Information Networking and Applications.IEEE,2012: 113119.

[7] Zhang Leiyuan, Shu Aiming. A Practical Method of Road Intersection Signal Phase Design [J]. Proceedings of the 7th China Intelligent Transportation Annual Excellent - Intelligent Transportation Technology,2012: 160-169.

[8] Gao Yunfeng, Chen Hongjie. Research on Optimization of Signal Phase and Phase Sequence at Isolated Intersection [J]. Proceedings of 2007 Annual Meeting of the Third China Intelligent Transportation,2007: 949-953.

[9] Webster FV. Traffic signal settings[R].1958.

[10] Wang X, Chang Y, Zhang P. Traffic signal optimization based on system equilibrium and bi-level multiobjective programing model[C]//nternational Conference on Green Intelligent Transportation System and Safety. Springer, Singapore, 2016: 429-438.

[11] Qu wenqiu, Zhang Chun, Zhang Hao, Huang Longyang, Li Chenglong. Study on Intersection Timing Optimization Based on VISSIM [J]. Electronic Test ,2021(12): 41-43.

[12] Liu Yating, Wang Fang. Environmental Protection Material Application in Traffic Engineering [J]. Anhui Architecture,2020,27(04): 190-192.

[13] Nie Sheng. Discussion on Environmental Protection Material Application in Traffic Engineering [J]. HEILONGJIANG JIAOTONG KEJI,2021,44(06): 176+178.

[14] Liu Zhixian. the Application of New Energy-saving Environmental Protection Material in Highway Engineering[J]. Green Environment Protection Building Materials, 2020(06): 24-25+27.

[15] Yuan Zhiyi. Shallow Discussion on the Application of Environmental Protection Concept in Traffic Engineering Construction [J]. Construction \& Design for Engineering,2017(14): 124-125.

[16] Road Marking Paint[J]. Technology and Market,2002(2): 9-9.

[17] Wen Hongjun, Hu Qinhu, Hao Shenqun. Application of Color Asphalt Pavement to a Non-motorized Vehicle Lane in Lanzhou[J]. CHINA MUNICIPAL ENGINEERING, 2008.

[18] Feng Qi. Discussion on the Advantages of Crumb Rubber Powder Modified Asphalt Surface Layer and Construction Control Points in Mountain Highways.

[19] Zhang Shuyan. Discussion on energy conservation and environmental protection measures on highways.

[20] Wang Huanxin. BASF Develops Energy-efficient, Environmentally Friendly Polyurethane Products and Applications[J].Guangzhou Chemical Industry 2011,01(1):1-1.

[21] Li Keping, et al. Code for Signal Control of Intersections on Urban Roads[S].DB 34/T 2423-2015. 